\title{
ANALYSIS OF PRACTICAL STABILITY FOR TIME DELAY AND DISTRIBUTED PARAMETER SYSTEMS
}

This paper points to sufficient conditions for practical stability of linear systems with time delay in state. Particularly, in control system engineering practice, despite the contribution to the contemporary control theory and system thinking, the problems of practical stability are not developed in details. Taking into account that the system can be stable in a classic way, but it can also possess inappropriate quality of dynamic behavior, and because of that, it is not applicable. For engineers it is crucial to take the system into consideration in relation to permitted states in phase space which are defined for such a problem, Dihovicni et al. (2006). Although, there are some papers covering practical stability problems, the lack of exploring it by using fundamental matrix and matrix measure was observed. Our main idea is to present definitions and conditions for practical stability, applying matrix measure approach. From a practical view point, it is crucial to find intervals on which the system is stable, and to know the function of initial state, the "prehistory" of system motion. The practical stability for a class of a distributed parameter system is also presented. The system is described in state space and a unique theory for such a problem is developed where a fundamental matrix of system and matrix measure is used. Using an efficient approach based on matrix measure and system fundamental matrix, the theorems for practical stability of distributed parameter systems are developed, and superiority of our results is illustrated with a numerical example.

Keywords: distributed parameter systems, time delay, practical stability.

\section{Introduction}

During the process of analysis and synthesis of control systems, the fundamental problem is stability, Wang (1992). From strictly engineering point of view it is very important to know the boundaries where a system trajectory comes during the motion in state space. The most often case for consideration of control systems, is behaving on an infinite interval which in real cases is only of academic importance in spite of practical stability which has significance in real life, Nedic et al. (2006). The linear systems might have delay in state, in control, or in both of them, but the most frequent occurring delay is in state, Debeljkovic (1994). Considering engineering practice needs, it is important to explore the time intervals where the system is stable. Thus, the development of efficient theory is essential for necessary and sufficient conditions for practical stability, Nedic et al. (2006). In Tissir et al. (1996) are given conditions for practical stability, making relation between the matrix and its maximal and minimal singular values. Most authors focus on solving practical stability for modified Ljapunov concepts, according to Dihovicni et al. (2006). This paper contradicts the majority of theories dealing with this topic by developing a unique theory in state space using a fundamental matrix and matrix measure approach combined with conditions of singular values of matrix $A_{0}$ and $A_{1}$.

\footnotetext{
* Djordje N. Dihovicni

Technical College, New Belgrade, Serbia, E-mail: ddihovic@ Eunet.yu
}

\section{General Consideration}

Consider the time delay system described by:

$$
\underline{\dot{x}}=A_{0} \cdot \underline{x}(t)+A_{1} \cdot \underline{x}(t-\tau)
$$

with an appropriate function of initial state:

$$
x(t)=\underline{\psi}_{x}(t)-\tau \leq t \leq 0
$$

where the system described by equation (1) is presented in a free working state, $\underline{x}(t)$ is the state vector, and $A_{0}$ and $A_{1}$ are constants of the system matrix of appropriate dimension, and $\tau$ is time delay.

The system behavior described by equation (1) is defined on time interval, $J=\left\{t_{0}, t_{0}+T\right\}$, Nenadic et al. (1997), and $T$ can be a positive real number.

\section{Main Result}

Theorem 1: Time delay system (1) with a function of initial state (2), is stable on a finite time interval in relation with $[\alpha, \beta$, $\tau, T]$ if the following conditions are satisfied : 
a) $\left\|A_{1}\right\|<\frac{\sigma_{\max }\left(Q^{1 / 2}\right)}{\sigma_{\max }\left(Q^{-1 / 2} P\right)}$

where $P$ is a solution of matrix equation:

$$
A_{0}^{T} \cdot P+P \cdot A_{0}=-2 \cdot Q
$$

and $\sigma_{\max }\left(A_{0}\right)$ and $\sigma_{\min }\left(A_{0}\right)$, are maximal and minimal singular values of matrix $A_{0}$, and holds:

$$
\begin{aligned}
& \left\|A_{1}\right\|<\frac{\sigma_{\max }\left(Q^{1 / 2}\right)}{\sigma_{\max }\left(Q^{-1 / 2} P\right)} \\
& \text { b) } e^{\mu\left(A_{0}\right) t}<\frac{\sqrt{\beta / \alpha}}{1+\tau\left\|A_{1}\right\|}, \forall t \in[0, T]
\end{aligned}
$$

Proof of conditions a): Consider the functional, presented in Tissir et al. (1996):

$$
\begin{aligned}
& v=\underline{x}^{T}(t) \cdot P \cdot \underline{x}(t)+\int_{t-\tau}^{t} \underline{x}^{T}(s) \cdot Q \cdot \underline{x}(s) \cdot d s \\
& \dot{v}(t)=\underline{x}^{T}(t) \cdot\left(A_{0}^{T} \cdot P+P \cdot A_{0}+Q\right) \cdot \underline{x}(t)+2 \cdot \underline{x}^{T}(t) \\
& P \cdot A_{1} \cdot \underline{x}(t-\tau)-\underline{x}^{T}(t-\tau) \cdot Q \cdot \underline{x}(t-\tau) \\
&= \underline{x}^{T}(t) \cdot\left(A_{0}^{T} \cdot P+P \cdot A_{0}+2 \cdot Q\right) \cdot \underline{x}(t) \cdot Q \cdot \underline{x}(t)+(8) \\
&+2 \cdot \underline{x}^{T}(t) \cdot P \cdot A_{1} \cdot \underline{x}(t-\tau)-\underline{x}^{T}(t-\tau) \\
& \cdot Q \cdot \underline{x}(t-\tau)
\end{aligned}
$$

From equation (4) and the inequality:

$$
\begin{aligned}
& 2 \cdot \underline{x}^{T}(t) \cdot P \cdot A_{1} \cdot \underline{x}(t-\tau)= \\
& =2 \cdot \underline{x}^{T}(t) \cdot P \cdot A_{1} \cdot Q^{-1 / 2} \cdot Q^{1 / 2} \cdot \underline{x}(t-\tau) \\
& \leq \underline{x} T(t) \cdot P \cdot A_{1} \cdot Q^{-1} \cdot A_{1}^{T} \cdot P \cdot \underline{x} T(t)+\underline{x}^{T}(t-\tau) \cdot Q \cdot \underline{x}(t-\tau)
\end{aligned}
$$

it is obtained:

$$
\begin{aligned}
\dot{v} & =-\underline{x}^{T}(t) \cdot Q \cdot \underline{x}(t)+2 \cdot \underline{x} \underline{x} \cdot P \cdot A_{1} \cdot \underline{x}(t-\tau)-\underline{x}^{T}(t-\tau) \cdot Q \cdot \underline{x}(t-\tau) \\
& \leq-\underline{x}^{T}(t) \cdot Q \cdot \underline{x}(t)+\underline{x}^{T}(t) \cdot P \cdot A_{1} \cdot Q^{-1} \cdot A_{1}^{T} \cdot P \cdot \underline{x}(t) \\
& \leq-\underline{x^{T}}(t) \cdot Q^{1 / 2} \cdot\left(t-Q^{-1 / 2} \cdot P \cdot A_{1} \cdot Q^{-1} \cdot A_{1}^{T} \cdot P \cdot Q^{-1 / 2}\right) \cdot Q^{-1 / 2} \cdot \underline{x}(t)
\end{aligned}
$$

where $\dot{v}(t)$ is negative definite if the following equation holds:

$$
1-\lambda_{\max }\left(Q^{-1 / 2} \cdot P \cdot A_{1} \cdot Q^{-1} \cdot A_{1}^{T} \cdot P \cdot Q^{-1 / 2}\right)>0
$$

which is satisfied if the following equation holds:

$$
1-\sigma_{\max }^{2}\left(Q^{-1 / 2} \cdot P \cdot A_{1} \cdot Q^{-1 / 2}\right)>0
$$
if:

Using the properties, Amir-Moez (1956), condition (10) holds 72 COMMUNICATIONS 3/2011

$$
1-\sigma_{\max }^{2}\left(Q^{-1 / 2} \cdot P\right) \cdot \sigma_{\max }^{2}\left(A_{1} \cdot Q^{-1 / 2}\right)>0
$$

which is satisfied if:

$$
1-\frac{\left\|A_{1}\right\|^{2} \cdot \sigma_{\max }^{2}\left(Q^{-1 / 2} \cdot P\right)}{\sigma_{\min }^{2}\left(Q^{1 / 2}\right)}>0
$$

and the proof is complete.

Using a similar approach, Nenadic et al. (1997):

$$
\left|\int_{a}^{b} \varphi(x) d x\right| \leq \int_{a}^{b}|\varphi(x)| \cdot d x
$$

it is obtained:

$$
\begin{aligned}
\underline{a}^{T}(t) \underline{c}(t) & \leq\left|\underline{\psi}_{x}^{T}(0) \Phi^{T} \int_{\tau}^{0} \underline{b}(t, \theta) d \theta\right| \leq\left\|\underline{\psi}_{x}^{T}(0) \Phi^{T}(t)\right\|\left\|_{\sigma_{\tau}}^{0} \underline{b}(t, \theta) d \theta\right\| \\
& \leq\left\|\underline{\psi}_{r}^{T}(0)\right\| \Phi^{T}(t)\|\|_{\int_{\tau}} \underline{b}(t, \theta) \| d \theta
\end{aligned}
$$

and equation (16) can be written as:

$$
\begin{aligned}
& \underline{x}^{T}(t) \underline{x}(t)=\underline{\psi}_{x}^{T}(0) \cdot \Phi^{T}(t) \cdot \Phi(t) \cdot \underline{\psi}_{x}(0)+2 \cdot\left\|\underline{\psi}_{x}^{T}(0)\right\| \cdot\left\|\Phi^{T}(t)\right\| . \\
& \cdot \int_{-\tau}^{0}\|\Phi(t-\theta-\tau)\|\left\|A_{1}\right\|\left\|\underline{\psi}_{r}(\theta)\right\| \cdot d \theta+\left\|\int_{-\tau}^{0} \underline{b}(t, \theta) d \theta\right\|
\end{aligned}
$$

or:

$$
\begin{aligned}
& \underline{x}^{T}(t) \underline{x}(t)=\underline{\psi}_{x}^{T}(0) \cdot \Phi^{T}(t) \cdot \Phi(t) \cdot \underline{\psi}_{x}(0)+2 \cdot\left\|\underline{\psi}_{x}^{T}(0)\right\| \cdot\left\|\Phi^{T}(t)\right\| . \\
& \cdot \int_{-\tau}^{0}\|\Phi(t-\theta-\tau)\| A_{1}\|\| \underline{\psi}_{x}(\theta) \| \cdot d \theta+\left[\int_{-\tau}^{0}\|\Phi(t-\theta-\tau)\| A_{1}\left\|\underline{\Psi}_{x}(\theta)\right\| \cdot d \theta\right]^{2}
\end{aligned}
$$

If we use the well known relation:

$$
m(t) \leq\|\Phi(t-\theta-\tau)\| \cdot\left\|\underline{\psi}_{x}(\theta)\right\| \leq M(t), \forall \theta \in[-\tau, 0]
$$

then it yields:

$$
m(t) \cdot \tau \leq\|\Phi(t-\theta-\tau)\| \cdot\left\|\underline{\psi}_{x}(\theta)\right\| \leq M(t) \cdot \tau
$$

It is possible to show that it yields:

$$
\|\Phi(t-\theta-\tau)\|_{\theta \in[-\tau, 0]} \leq\|\Phi(t)\|,\left\|\underline{\psi}_{x}(\theta)\right\|_{\theta \in[-\tau, 0]}<\sqrt{\alpha}
$$

and:

$$
\left\|\underline{\psi}_{x}(0)\right\|<\sqrt{\alpha},\left\|\Phi^{T}(t)\right\|=\|\Phi(t)\|
$$

so the equation (18) can be shown as:

$$
\begin{gathered}
\underline{x}^{T}(t) \cdot \underline{x}(t) \leq\|\Phi(t)\|^{2} \underline{\psi}_{x}^{T}(0) \underline{\psi}_{x}(0)+2 \cdot\left\|\underline{\psi}_{r}^{T}(0)\right\| \cdot\left\|\Phi^{T}(t)\right\| \cdot\left\|A_{1}\right\| \cdot \tau \cdot \sqrt{\alpha} \\
+\left\|\Phi^{T}(t)\right\|^{2} \cdot\left\|A_{1}\right\|^{2} \cdot \tau^{2} \cdot \alpha
\end{gathered}
$$

and if we use the well-known inequality, it is given:

$$
\|\Phi(t)\|=\left\|\exp \left[A_{0}(t)\right]\right\| \leq \exp \left\{\mu\left(A_{0}\right) \cdot t\right\}
$$

where $\mu$ is defined as: 


$$
\mu\left(A_{0}\right)=\frac{1}{2} \cdot \lambda_{\text {max }}\left(A^{T}+A\right)
$$

and if we use the following inequality:

$$
\underline{\psi}_{r}^{T}(0) \cdot \underline{\psi}_{r}(0)<\alpha\left(\left\|\underline{\psi}_{r}^{T}(0)\right\|=\left\|\underline{\psi}_{r}(0)\right\|<\sqrt{\alpha}\right)
$$

then it yields:

$$
\begin{aligned}
& \underline{x}^{T}(t) \cdot \underline{x}(t) \leq e^{2 \mu\left(A_{0}\right) \cdot t} \cdot \alpha+2 \cdot e^{2 \mu\left(A_{0}\right) t}\left\|A_{1}\right\| \cdot \tau \cdot \alpha \\
& \quad+2 \cdot e^{2 \mu\left(A_{0}\right) \cdot t}\left\|A_{1}\right\|^{2} \cdot \tau^{2} \cdot \alpha=e^{2 \mu\left(A_{0}\right) \cdot t} \alpha\left(1+\tau\left\|A_{1}\right\|\right)^{2}
\end{aligned}
$$

If we apply the theorem basic condition given by equation (5) to the previous inequality, then it is obtained:

$$
\underline{x}^{T}(t) \cdot \underline{x}(t)<\left(\frac{\sqrt{\beta / \alpha}}{1+\tau \cdot\left\|A_{1}\right\|}\right) \cdot \alpha\left(1+\tau \cdot\left\|A_{1}\right\|\right)^{2}<\beta
$$

and thus the proof is complete.

Theorem 2: Time delay system (1) with the function of initial state (2) is stable on finite time interval in relation with $[\alpha, \beta, \tau, T$, $\mu_{2}\left(A_{0}\right) \neq 0$ ], if the following conditions are satisfied:

a) $\operatorname{Re}\left[\lambda_{i}\left(A+A_{1} \cdot e^{-s \cdot \tau}\right)\right]<0 \quad i=1$,É,$n$

B) $e^{\mu_{2}\left(A_{0}\right) t}<\frac{\beta / \alpha}{1+\mu_{2}^{-1}\left(A_{0}\right) \cdot\left\|A_{1}\right\|_{2} \cdot\left(1-e^{-\mu_{2}\left(A_{0}\right) \tau}\right)}, \forall t \in[0, T]$ (30)

In Tissir et al. (1996) the proof of condition a is given.

Theorem 3: Time delay system (1) with the function of initial state (2), is stable on finite time interval in relation with $[\alpha, \beta, \tau$, $T$ ], if condition a) of theorem 2 is satisfied, and if the following condition is satisfied:

$$
1+t \cdot\left\|A_{1}\right\|_{2}<\beta / \alpha, \quad \forall t \in[0, \tau]
$$

Proof: It yields from theorem 2 when $\mu_{2}\left(A_{0}\right)=0$.

\section{Definitions and Conditions of Practical Stability}

Let us consider the first order hyperbolic distributed parameter system which is decribed by the following state-space equation

$$
\frac{\partial \underline{x}(t, z)}{\partial t}=A_{0} \cdot \underline{x}(t, z)+A_{1} \frac{\partial \underline{x}}{\partial z}
$$

with an appropriate function of initial state:

$$
\begin{aligned}
& \underline{x}_{0}(t, z)=\underline{\psi}_{x}(t, z) \\
& 0 \leq t \leq \tau, 0 \leq t \leq \zeta
\end{aligned}
$$

where $\underline{x}(t, z)$ is $\mathrm{n}$-component real vector of system state, $\mathrm{A}$ is the matrix appropriate dimension, $\mathrm{t}$ is time and $\mathrm{z}$ is the space coordinate.
Definition 1: The distributed parameter system described by equation (32) that satisfies the initial condition (33) is stable on finite time interval in relation to $[\xi(t, z), \beta, T, Z]$ if and only if:

$$
\begin{aligned}
& \underline{\psi}_{x}^{T}(t, z) \cdot \underline{\psi}_{x}(t, z)<\xi(t, z) \\
& \forall t \in[0, \tau], \forall z \in[0, s]
\end{aligned}
$$

then it follows:

$$
\begin{aligned}
& \left.\underline{x}^{T}(t, z)\right) \cdot \underline{x}(t, z)<\beta \\
& \forall t \in[0, T], \forall z \in[0, Z]
\end{aligned}
$$

where $\xi(t, z)$ is the scalar function with feature $0<\xi(t, z) \leq \alpha$, $0 \leq t \leq \tau, 0 \leq z \leq \zeta$ where $\alpha$ is a real number, $\beta \in R$ and $\beta>\alpha$.

Let us calculate the fundamental matrix for this class of system:

$$
\frac{d \Phi(s, \sigma)}{d \sigma}=A_{1} \cdot(s I-A) \cdot \Phi(s, \sigma)
$$

where after double Laplace transformation and necessary approximation it yields:

$$
\Phi(t, z)=\exp (A \cdot t \cdot z)
$$

where $A=\frac{I-A_{0} \cdot A_{1}}{A_{1}}$.

Theorem4: The distributed parameter system described by equation (32) that satisfies the internal condition (33) is stable on finite time interval in relation to $[\xi(t, z), \beta, T, Z]$ if and only if the following condition is satisfied:

$$
e^{2 \mu(A) \cdot t z}<\frac{\beta}{\alpha}
$$

Proof: The solution of equation (32) with the initial condition (33) can be described as:

$$
\underline{x}(t, z)=\Phi(t, z) \cdot \underline{\psi}(0,0)
$$

Using the above equation it follows:

$$
\begin{array}{r}
\underline{x}^{T}(t, z) \cdot \underline{x}^{T}(t, z)=\left[\underline{\psi}_{x}^{T}(0,0) \cdot \Phi(t, z)\right] \\
{\left[\underline{\psi}_{x}^{T}(0,0) \cdot \Phi(t, z)\right]}
\end{array}
$$

Using the well-known ineqality:

$$
\|\Phi(t, z)\|=\|\exp [A \cdot t \cdot z]\| \leq \exp \{\mu(A) \cdot t \cdot z\}
$$

and taking into account that:

$$
\begin{aligned}
& \underline{\psi}_{x}^{T}(0,0) \cdot \underline{\psi}_{x}(0,0)<\alpha \\
& \left(\left\|\underline{\psi}_{x}^{T}(0,0)\right\|=\left\|\underline{\psi}_{x}^{T}(0,0)\right\|<\alpha\right)
\end{aligned}
$$


then it follows:

$$
\underline{x}^{T}(t, z) \cdot \underline{x}(t, z) \leq e^{2 \mu(A \cdot t z)} \cdot \alpha
$$

Applying the basic condition of theorem 1 by using equation (38) to further inequality the following is obtained, Dihovicni et al. [4]:

$$
\underline{x}^{T}(t, z) \cdot \underline{x}(t, z)<\left(\frac{\beta}{\alpha}\right) \cdot \alpha<\beta
$$

Theorem 5: The distributed parameter system described by equation (32) that satisfied the initial condition (33) is stable on finite time interval in relation to $[\xi(t, z), \beta, T, Z]$ if the following condition is satisfied:

$$
\begin{aligned}
& e^{\mu(A) t z z}<\frac{\sqrt{\beta / \alpha}}{1+\tau \cdot \zeta\|A\|} \\
& \forall t \in[0, \tau], \forall z \in[0, S]
\end{aligned}
$$

The proof of this theorem is given in Dihovicni et al. [3].

Let $|\underline{x}|_{(\cdot)}$ be any vector norm and any matrix norm $\|\cdot\|_{2}$ which originated from this vector. Following expresions are used:

$$
|\underline{x}|_{2}=\left(\underline{x}^{T} \cdot \underline{x}\right)^{1 / 2} \text { and }\|\cdot\|_{2}=\lambda_{\max }^{1 / 2}\left(A^{*} \cdot A\right)
$$

where * and $T$ are transpose-conjugate and transport matrixes.

It is important to define the matrix measure as:

$$
\begin{aligned}
& \mu(A)=\lim _{\varepsilon \rightarrow 0} \frac{\|1+\varepsilon \cdot A\|-1}{\varepsilon} \\
& \mu_{1}(A)=\max \left(\operatorname{Re}\left(a_{k k}\right)+\sum_{i=1, i \neq k}^{n}\left|a_{i k}\right|\right) \\
& \mu_{2}(A)=\frac{1}{2} \max \lambda_{i}\left(A^{T}+A\right) \\
& \mu_{\infty}(A)=\max \left(\operatorname{Re}\left(a_{i i}\right)+\sum_{k=1}^{n}\left|a_{k i}\right|\right)
\end{aligned}
$$

Definition 2: The distributed parameter system described by equation (32) that satisfies the initial condition (33) is stable on finite time interval in relation to $[\xi(t, z), \beta, T, Z]$ if and only if, Dihovicni et al. [3]:

$$
\left|\underline{\psi_{x}(t, z)}\right|<\xi(t, z)
$$

then it follows:

$$
|\underline{x}(t)|_{2}<\beta
$$

where $\xi(t, z)$ is scalar function with feature $0<\xi(t, z) \leq \alpha$, $0 \leq t \leq \tau, 0 \leq z \leq \zeta) \alpha$ is real number, $\beta \in R$ and $\beta>\alpha$.

Theorem 6: The distributed parameter system described by equation (32) that satisfies the initial condition (33) is stable on finite time interval in relation to $[\alpha, \beta, T, Z]$ if and only if the following condition is satisfied:

$$
\begin{aligned}
& e^{\mu_{2}(A) \cdot t z}<\frac{\sqrt{\beta / \alpha}}{1+\mu^{-1}{ }_{2}(A)} \\
& \forall t \in[0, T], \forall z \in[0, Z]
\end{aligned}
$$

Proof: The solution of equation (1) with the initial condition (2) can be described by the fundamental matrix as:

$$
\underline{x}^{x}(t, z)=\Phi(t, z) \cdot \underline{\psi}_{x}(0,0)
$$

Using the norms of the left and right side of the equation (51) it follows:

$$
\underline{x}^{T}(t, z) \cdot \underline{x}(t, z) \leq e^{2 \mu(A \cdot t \cdot z)} \cdot \alpha
$$

and using the well-known inequality:

$$
\|\exp (A \cdot t \cdot z)\|_{2} \leq \exp \{\mu(A \cdot t \cdot z)\}
$$

$$
t \geq 0, \quad z \geq 0
$$

it follows:

$$
|\underline{x}(t, z)|_{2} \leq e^{\mu_{2}(A) t \cdot z}\left|\underline{\psi_{x}(0,0)}\right|_{2}
$$

and using equation (49) the following is obtained:

$$
|\underline{x}(t, z)|_{2} \leq \alpha \cdot e^{\mu_{2}(A) \cdot t z}
$$

so finally the following is obtained:

$$
|\underline{x}(t, z)|_{2} \leq \alpha \cdot e^{\mu_{2}(A) t \cdot z}\left\{1+\mu_{2}^{-1}(a)\right\}
$$

Applying the basic condition of theorem 3 by using equation (50) the following is obtained:

$$
\begin{aligned}
& |\underline{x}(t)|_{2}<\beta \\
& \forall t \in[0, T], \forall z \in[0, Z]
\end{aligned}
$$

Theorem 7: The distributed parameter system described by equation (32) that satisfies the initial condition (33) is stable on finite time interval in relation to $[\alpha, \beta, T, Z]$, if and only if the following condition is satisfied, Dihovicni et al. [3]:

$$
\begin{aligned}
& e^{\mu(A+z)}<\frac{\beta}{\alpha} \\
& \forall t \in[0, T], \forall z \in[0, Z]
\end{aligned}
$$

Theorem 8: The distributed parameter system described by equation (1) that satisfies the initial condition (2) is stable on finite time interval in relation to $\left[t_{0}, J, \alpha, \beta, Z\right]$, if and only if the following condition is satisfied: 


$$
\begin{aligned}
& {\left[1+\left(t-t_{0}\right) \cdot \sigma_{\max }\right]^{2} \cdot e^{2\left(t-t_{0}\right) z \cdot \sigma_{\max }}<\frac{\beta}{\alpha},} \\
& \forall t \in[0, J], \forall z \in[0, Z]
\end{aligned}
$$

where $\sigma_{\max }$ represents the maximum singular value of matrix. The proof of this theorem is given in Dihovicni et al. [4].

\section{Example}

Consider the distributed parameter system:

$$
\frac{\partial \underline{x}(t, z)}{\partial t}=\left[\begin{array}{cc}
2 & 1 \\
-2 & 4
\end{array}\right] \frac{\partial \underline{x}}{\partial z}+\left[\begin{array}{cc}
1 & 0 \\
2 & -1
\end{array}\right] \underline{x}(t, z)
$$

where

$$
A_{0}=\left[\begin{array}{cc}
1 & 0 \\
2 & -1
\end{array}\right], A_{1}=\left[\begin{array}{cc}
2 & 1 \\
-2 & 4
\end{array}\right]
$$

and according to equation (47) it is obtained:

$$
A=\left[\begin{array}{cc}
0.548 & 0.904 \\
0.1653 & 3.320
\end{array}\right]
$$

with the initial condition:

$$
\underline{x}_{0}(t, z)=\underline{\psi}_{x}(t)=\left[\begin{array}{ll}
0 & 1
\end{array}\right]^{T}
$$

Let us assume the following values:

$$
\alpha=2.0 ; \beta=60.22 ; \kappa_{\text {est }}=0.7 \mathrm{~s}
$$

where $\mathrm{\kappa}=t \cdot z$, and by using initial condition

$$
\left\|\underline{\psi}_{x}\right\|^{2}=1<2.01
$$

Let us calculate the matrix measure $\mu$ and the norm:

$$
\begin{aligned}
& \mu(A)=3.37 \\
& \|A\|=3.45
\end{aligned}
$$

Combining this values into equation (7), it yields:

$$
\begin{aligned}
& e^{2 \cdot t z}<29.96 \\
& \forall t \in[0, T], z \in[0,1]
\end{aligned}
$$

with solution:

$$
\kappa_{\text {est }}=1.69 \mathrm{~s}
$$

Upon theorem 5 it yields:

$$
\begin{aligned}
& e^{t \cdot s}<1.23 \\
& \forall t \in[0, T], z \in[0,1]
\end{aligned}
$$

with solution:

$$
\kappa_{e s t}=0.207 \mathrm{~s}
$$

Using theorem 6 it follows:

$$
e^{t \cdot s}<23.31
$$

whose solution is:

$$
\kappa_{e s t}=3.14 s
$$

Upon theorem 7 it yields:

$$
e^{t \cdot s}<29.96
$$

and solution is:

$$
\kappa_{e s t}=3.39 s
$$

If we use theorem 5 then it is obtained:

$$
[1+3.45 t]^{2} \cdot e^{2 \cdot 3.45 \cdot t z}<29.96
$$

with solution:

$$
\kappa_{e s t}=0.06 s
$$

Analysing the values for estimation it is obvious that the best result is obtained using theorem 5 .

\section{Conclusion}

During the analyse the process systems from safety and optimal cost perspective, it is important to recognize which systems are not stable in real conditions. From engineering point of view we are interested in such systems which are stable in finite periods of time. Therefore, our first concern should be to maintain stable and safe systems.

Taking into account the principle of practical stability, the following conditions must be satisfied:

- determine the set $S_{\beta-}$ find the borders for the system motion;

- determine the set $S_{\varepsilon-}$ find maximum amplitudes of possible disturbance;

- determine the set $S_{\alpha}$ of all initial state values.

In case that these conditions are regularly determined it is possible to analyse the system stability from a practical stability view point.

The main conclusion from the result for distributed parameter systems is that the estimated interval which is determined by previously explained theorems and which represents the product of tz for this class of distributed parameter systems is very near to the real value. Therefore, the theory is applicable and has a widespread use in determination where the system is stable. 


\section{COMMNICOIIONS}

\section{References}

[1] TOKASHIKI, L.R, FUJITA, T., KAGAWA, T., PAN, W.: Dynamic Characteristics of Pneumatic Cylinders Including Pipes, $9^{\text {th }}$ Bath Int'IFluid Power Workshop, 1996, pp 1-14.

[2] DIHOVICNI, N. DJ., NEDIC, N.: Stability of Distributed Parameter Systems on Finite Space Interval, 32end Yupiter Conference, Zlatibor, 2006, pp 306-312.

[3] DIHOVICNI, N.DJ., NEDIC, N.: Stability of Distributed Parameter Systems on Finite Space Interval described by cubic equations, 32 end Yupiter Conference, Zlatibor, 2006, pp 321-325.

[4] DIHOVICNI, N.DJ., NEDIC, N.: Practical Stability of Linear Systems with Delay in State, AMSE, Association for the Advancement of Modelling \& Simulation Techniques in Enterprises, Tassin La-Demi-Lune, France, Vol. 62, No. 2, 2007, pp 98-104.

[5] NOVAKOVIC, B.: Control Methods of Technical System, Skolska knjiga, Zagreb 1989.

[6] ERNAT, J., GILLES, D.: A Systeme mit Verteilten Parametern, (Book style), Wien 1973.

[7] BROWN, F.T.: The Transient Response of Fluid Lines, J. of Basic Engineering, Trans of ASME, Series D, 84-4, 547/553, 1962.

[8] KagAwA, T., FUJITA, T., TAKEUCHI, M.: Dynamic Response and Simulation Model of Pneumatic Pipe Systems, Proc. of $7^{\text {th }}$ Bath International Fluid Power Workshop, 1994. 